This item was submitted to Loughborough's Research Repository by the author.

Items in Figshare are protected by copyright, with all rights reserved, unless otherwise indicated.

\title{
Spatio-temporal monitoring of wheat yellow rust using UAV multispectral imagery
}

\section{PLEASE CITE THE PUBLISHED VERSION}

\section{PUBLISHER}

Elsevier

\section{VERSION}

AM (Accepted Manuscript)

\section{PUBLISHER STATEMENT}

This paper was accepted for publication in the journal Computers and Electronics in Agriculture and the definitive published version is available at https://doi.org/10.1016/j.compag.2019.105035.

\section{LICENCE}

CC BY-NC-ND 4.0

\section{REPOSITORY RECORD}

Su, Jinya, Cunjia Liu, Xiaoping Hu, Xiangming Xu, Lei Guo, and Wen-Hua Chen. 2019. "Spatio-temporal Monitoring of Wheat Yellow Rust Using UAV Multispectral Imagery". figshare.

https://hdl.handle.net/2134/9926888. 


\title{
Spatio-temporal monitoring of wheat yellow rust using UAV multispectral imagery
}

\author{
Jinya $\mathrm{Su}^{\mathrm{a}, *}$, Cunjia Liu ${ }^{\mathrm{b}}$, Xiaoping $\mathrm{Hu}^{\mathrm{c}, *}$, Xiangming $\mathrm{Xu}^{\mathrm{d}}$, Lei Guo ${ }^{\mathrm{e}}$, Wen-Hua Chen ${ }^{\mathrm{b}}$ \\ ${ }^{a}$ School of Computer Science and Electronic Engineering, University of Essex, Colchester, CO4 $3 S Q$, U.K. \\ ${ }^{b}$ Department of Aeronautical and Automotive Engineering, Loughborough University, Loughborough, LE11 $3 T U$, U.K. \\ ${ }^{c}$ State Key Laboratory of Crop Stress Biology for Arid Areas, College of Plant Protection, Northwest A\&F University, Yangling, China. \\ ${ }^{d}$ Department of Pest \&S Pathogen Ecology, NIAB EMR, West Malling, ME19 6BJ, U.K. \\ e School of Automation Science and Electrical Engineering, Beihang University, Beijing 100191, China
}

\begin{abstract}
This work is focused on the spatio-temporal monitoring of winter wheat inoculated with various levels of yellow rust inoculum during the entire growth season. A dedicated workflow is devised to obtain time-series five-bands (visible-infrared) aerial imageries with a multispectral camera and an Unmanned Aerial Vehicle. A number of spectral indices are drawn so that the sensitive ones can be identified by statistical dependency analysis; particularly, their discriminating capabilities are evaluated at different stages for both wheat pixel segmentation and yellow rust severity. Then the spatial-temporal changes of sensitive bands/indices are evaluated and analysed quantitatively. A validation field experiment was designed in 2017-2018 by inoculating wheat with one of the six levels of yellow rust inoculum. Five-bands RedEdge camera on-board DJI S1000 was used to capture aerial images at eight time points covering the entire growth season at an altitude of about 20 meters with a ground resolution of 1-1.5 cm/pixel. Experimental results via spatio-temporal analysis show that: (1) various bands/indices should be used for wheat segmentation at different stages; (2) no bands/indices differences are observed for yellow rust inoculated wheat plots in both incubation stage (9 days after inoculation) and early onset stage (25 days after inoculation); (3) NIR and Red are the sensitive bands for wheat yellow rust in disease stages (45 days after inoculation); and their normalized difference NDVI index provides an even higher statistical dependency; (4) bands/indices' sensitivity to yellow rust changes over time and decreases in later Heading stage until being very low in Ripening stage (61 days after inoculation). This experimental study provides a crucial guidance for future early spatio-temporal yellow rust monitoring at farmland scales.
\end{abstract}

Keywords: Multispectral image; Spatio-temporal analysis; Statistical dependency; UAV remote sensing; Yellow rust

\section{Introduction}

Wheat is the most widely grown crop in the world; their demand is expected to increase by $60 \%$ with a predicted world population of 9 billion in 2050. However, wheat is vulnerable to a number of diseases and pests such as yellow rust, fusarium head blight, sharp eyespot, powdery mildew, aphid, etc. causing significant losses to farmers and

\footnotetext{
* Joint corresponding authors

Email addresses: j.su@essex.ac.uk (Jinya Su), C.Liu5@lboro.ac.uk (Cunjia Liu), xphu@nwsuaf.edu.cn (Xiaoping Hu), Xiangming.Xu@emr.ac.uk (Xiangming Xu), lguo@buaa.edu.cn (Lei Guo), W.Chen@lboro.ac.uk (Wen-Hua Chen)
} 
threatening food security if not being controlled properly [1]. Timely, effective and precise disease control for wheat crop is, therefore, of paramount importance to meet the world's future food security and sustainability needs.

Conventional calendar-scheduled pesticide application for disease control generally does not consider disease development and its spatial distribution, often leading to excessive use of pesticides and the associated harmful effects 2. Nowadays, decision-based crop management is gaining increasing research interests, where an automated and non-destructive spatio-temporal crop monitoring is a prerequisite, enabling the early and precise disease control [3]. Considering the high cost and time/labour intensity in manual spatio-temporal information acquisition and processing, low-altitude remote sensing technology is drawing increasing attention since it offers a practical (in terms of time, effort and cost) solution to monitor the spatio-temporal status of crop canopy at field scales [4, 5, 6] Particularly, in recent years Unmanned Aerial Vehicle (UAV) remote sensing with a user-defined spatial-temporal resolution and a high flexibility is gaining a lot of popularity, and has gradually become an important complement for satellite and manned-aircraft based remote sensing [7].

This work is, therefore, focused on the spatio-temporal monitoring of winter wheat inoculated with various levels of yellow rust inoculum by using a small UAV and a state-of-the-art multispectral camera. The yellow rust disease information can be applied to different applications such as providing map for site-specific application of fungicides, assessing the resistance of various wheat species to rust disease, etc. Wheat yellow (or stripe) rust, caused by Puccinia striiformis f. sp. tritici (Pst), is a common and destructive wheat disease worldwide, especially for regions with cool climates. It may cause a substantial yield loss, especially when environmental conditions are favourable (e.g. a temperatures of $5^{\circ} \mathrm{C}-15^{\circ} \mathrm{C}$, a moderate spring precipitation and a suitable wind direction for its spread). Yield losses can be reduced by timely fungicide application at the early stage of disease development, and therefore a precise spatio-temporal monitoring for yellow rust disease is crucial for its management.

Yellow rust disease inevitably results in certain physical and chemical changes on wheat leaves such as a reduction of ChlorophyII content, a water loss due to an increased evaporation, and visual rust symptoms on wheat leaves. In terms of visual symptom, it leads to the production of pustules that contain thousands of dry yellow-orange (early stage) to reddish-brown (late stage) spores, leading to the breakdown of pigments [8]. The symptom will result in certain changes in visible and NIR spectral ranges. Therefore, spectral sensors can be adopted to capture and monitor these changes via their spectral bands/wavelengths or defined indices [5].

Until now, most studies on yellow rust (or other diseases) monitoring are focused on the leaf scale by using ground level (or proximal sensing) methods via hyperspectral camera [1, 9], spectrograph [2], spectroscopy [10], spectrometer [11, 12, 13] or spectrophotometer [14. In these pioneering studies, the suitable wavelengths and narrow-band Spectral Indices (SIs) for yellow rust have been initially investigated. For example, 543nm (Green), 630nm, 750nm and 861nm 
(NIR) are proved to be effective wavelengths [2] via in-field images by a spectrograph (460-900nm). Among twenty-two narrow-band SIs from a spectrograph (450-1000nm), narrow-band Normalized Difference Vegetation Index (NDVI) ( $R_{850}$ for NIR and $R_{680}$ for Red) [15, Green Index (GI) [16] and Anthocyanin Reflectance Index (ARI) 17] are shown to be sensitive [14. ARI and Transformed ChlorophyII Absorption and Reflectance Index (TCARI) [18] were also identified to be effective among ten indices in [11] by using a spectrophotometer (450-900nm). The authors in [19] developed yellow rust index by ranking hyperspectral wavelengths using RELIEF-F filter. Sensitive wavelength selection on ground is important, while remote sensing (relatively broad) bands/indices selection (in consideration of spatial resolution) is more important for large-scale applications. It is noted that very little published information on airborne or satellite remote sensing is available for yellow rust. In [20], narrow-band Photochemical Reflectance Index $P R I=\left(R_{531}-R_{570}\right) /\left(R_{531}+R_{570}\right)$, was proved to be highly correlated with rust disease severity by using airborne hyperspectral imaging. Satellite remote sensing was also adopted in [21, 22, for the large-scale damage evaluation caused by yellow rust disease. Very recently, there are also several studies on yellow rust monitoring with an UAV at field scales. For example, in [23], RGB camera on-board an UAV is adopted to monitor yellow rust disease at an altitude of 100 meters. It was shown that Red band is most effective among visible broad bands. Five-bands multispectral camera on-board an UAV is adopted in [5], it was shown that both Red and NIR bands are effective and their normalized difference NDVI provides a even better result. These studies, however, are mainly focused on one-time monitoring of spatial disease distribution for yellow rust at disease stages, the temporal disease dynamics has yet to be investigated. Temporal information is crucial for early disease (or stress) detection; this idea has recently be exploited in wheat agronomy and breeding trials [4, dynamic grain yield prediction [24] and wheat senescence rate evaluation [25], and should also be exploited for yellow rust monitoring. The following observations are drawn to highlight the main research motivations:

(1) There is an urgent need for the spatio-temporal monitoring of wheat yellow rust to enable its precision management at field scales;

(2) Existing studies on yellow rust monitoring are mainly focused on either ground-level wavelengths/indices selection or airborne/satellite remote sensing for damage evaluation, which are not suitable for monitoring at field scales due to either a limited coverage or a poor spatial resolution with a high operational complexity/cost [7];

(3) Spatial evaluation of yellow rust by using one-time monitoring is important [5, 23, however, a spatio-temporal monitoring is more desirable.

Therefore, this work aims to evaluate the utility of multispectral camera and low-altitude UAV remote sensing platform for the spatio-temporal monitoring of winter wheat inoculated with various levels of yellow rust inoculum. 
The specific objectives of this study are to (i) devise a dedicated workflow for crop spatio-temporal monitoring at field scales by using an Unmanned Aerial Vehicle (UAV) and a five-band multispectral camera; (ii) identify effective bands/indices for wheat segmentation at different wheat growth (rust development) stages; (iii) identity effective spectral bands/indices for estimating yellow rust severity over time; (iv) investigate the temporal changes of suitable bands/indices under various levels of yellow rust severity.

\section{Materials}

In this section, materials relevant to the research are introduced such as field experiments for yellow rust disease, image acquisition by a five-bands multispectral camera on-board an UAV, and ground yellow rust severity data measurement.

\subsection{Field experiments}

Field experiments were conducted during October/2017-June/2018 at Caoxinzhuang experimental station (Geographic coordinate information with latitude: $34^{\circ} 307^{\prime} N$, longitude: $108^{\circ} 090^{\prime} E$ and altitude: 499 m a.s.l.) of Northwest Agriculture and Forestry (A\&F) University, Yangling, Shanxi Province, China (please refer to Fig 1 for the geographic location). The experimental field was about $17 \mathrm{~m}$ wide and $70 \mathrm{~m}$ long. The climate information of the region and soil characteristics of the experimental field are referred to Section 2.2 of our preceding work [5].

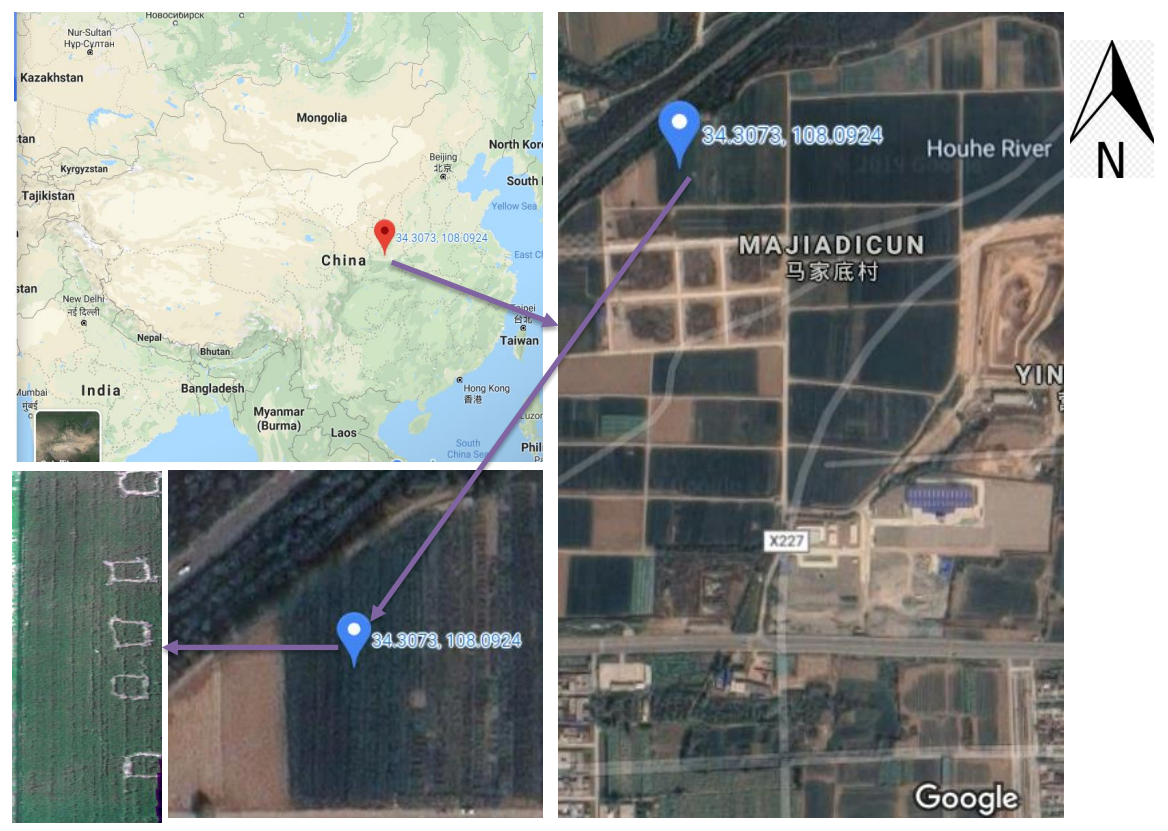

Figure 1: Location of the experimental wheat field.

Wheat variety Xiaoyan 22 was used in this study, which was highly susceptible to the race of yellow rust to be inoculated but moderately resistant to other diseases. Wheat seeds were sown on 05/Oct/2017 at a rate of 30 g 
seeds $/ \mathrm{m}^{2}$ and with a row spacing of about $16 \mathrm{~cm}$. As moisture is a vital factor for yellow rust disease development, the whole field was irrigated twice by the flooding method on 10/Dec/2017 and 13/Mar/2018. Wheat seedlings were inoculated on 23/Mar/2018 (before the jointing [elongation] stage) with the mixed Pst races (CYR 29, CYR 30, CYR 31, CYR 32, CYR 33), where the inoculation method was published previously [23]. In order to generate wheat plants infected with different levels of yellow rust, six square plots of $2 m \times 2 m$ (Fig 2), are inoculated with one of the six levels of yellow rust inoculum: $0,0.33 \mathrm{~g}, 0.67 \mathrm{~g}, 1.00 \mathrm{~g}, 1.33 \mathrm{~g}$ and $1.67 \mathrm{~g}$ (note: inoculation experiment with different levels of yellow rust inoculum was also done in the middle November of 2017 for the whole field, unfortunately, yellow rust symptom did not appear in Spring of 2018 due to low temperatures often below $-5^{\circ} \mathrm{C}$ in winter).

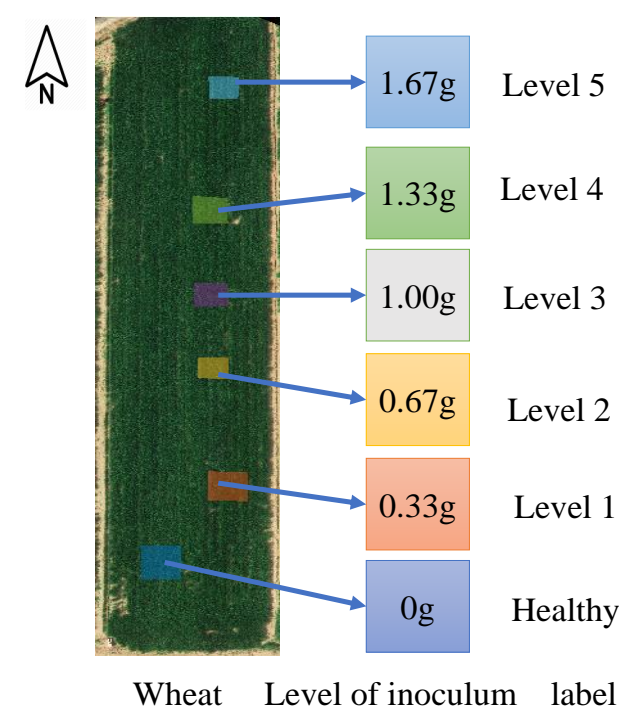

Figure 2: Wheat field (left) with six square plots being inoculated with one of the six levels of yellow rust inoculum (middle) and its corresponding label.

\subsection{Imaging by UAV-Multispectral camera system}

An UAV-camera system was developed to capture multispectral visible-infrared images for the wheat field at eight time points covering the entire wheat growth season (Table 1). The UAV-camera system (Fig 3) mainly consists of Spreading Wings S1000 Octocopter (DJI, Shenzhen, China) and the RedEdge multispectral camera (MicaSense Inc., Seattle, USA). In particular, DJI S1000 is a professional Octocopter with the following key parameters: height $(386 \mathrm{~mm})$, diameter $(1045 \mathrm{~mm})$, take-off weight $(6 \mathrm{~kg}-11 \mathrm{~kg})$ and hovering time (about 30 minutes). While RedEdge camera (weight: $135 \mathrm{~g}$, size: $5.9 \mathrm{~cm} \times 4.1 \mathrm{~cm} \times 3.0 \mathrm{~cm}$, resolution: $1280 \times 960$ pixels) is an off-the-shelf professional multispectral camera with five narrow bands including Blue, Green, Red, RedEdge and NIR. Their central wavelengths/bandwidth are 475nm\&20nm (Blue), 560nm\&20nm (Green), 668nm\&10nm (Red), 717nm\&10nm (RedEdge) and 840nm\&40nm (NIR), respectively. 


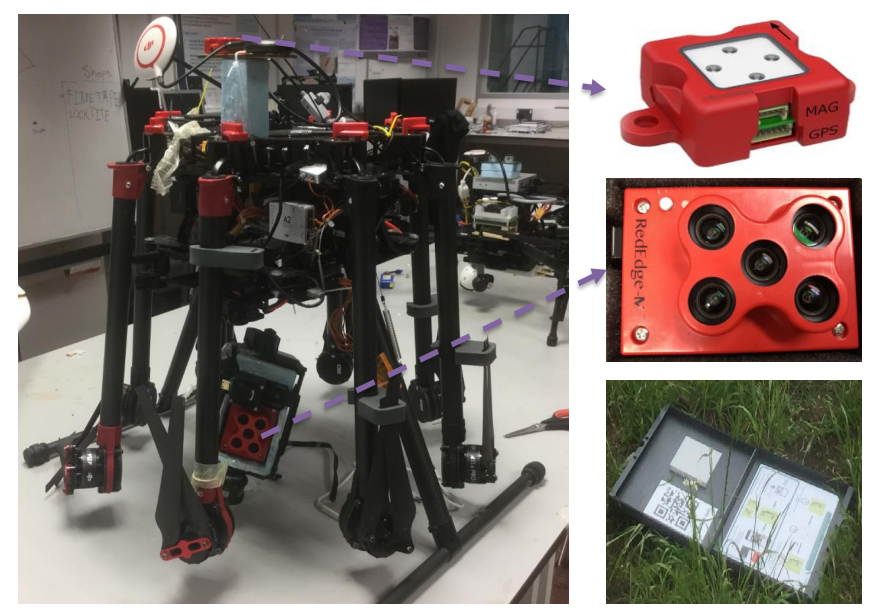

Figure 3: Image acquisition by an UAV-Camera system: DJI S1000 Octocopter (left), Downwelling light sensor (top-right), RedEdge camera (middle-right) and panel for reflectance calibration (bottom-right).

The spectral radiance values are influenced by many factors [26] such as time of measurement, sampling size, positioning of sensor, etc. In order to guarantee high-quality orthomosaic imageries for band reflectance and SI calculation, the following measures were taken: (i) a gimbal was adopted to fix the camera downward attenuating the adverse effects of UAV motion/vibration; (2) an overlap and sidelap of $75 \%$ was guaranteed by appropriately designing the flight path and UAV speed (i.e. $1 \mathrm{~m} / \mathrm{sec}$ ) by using DJI Ground Station 4.0, and camera triggering time (1 s interval); (3) the UAV imaging period is fixed between 11am-14pm of the day; (4) the altitude of UAV is controlled at a relatively low altitude (about 20 meters above ground) in comparison to previous studies of 50-100 meters [4, 23, as a result, the orthomosaic image has a relatively high ground sampling distance of $1-1.5 \mathrm{~cm} /$ pixel. While in order to appropriately calibrate aerial images, Downwelling Light Sensor (DLS) and MicaSense's Calibrated Reflectance Panel (CRP) were adopted concurrently. In particular, an image of the CRP was taken at an altitude of 1 meter before and after each flight. The details about UAV data acquisition during the entire wheat growth season are summarized in Table 1. In Table 1, the GSDs are different in various data collections, one can unify the resolution by resampling the image. This is not done in this work since the resolutions in the year of 2018 (in which year, yellow rust appeared) are very close and have little effects on the results according to empirical test.

\subsection{Ground yellow rust data collection}

In addition to UAV remote sensing images, ground assessment for yellow rust severity was also conducted. The ground yellow rust data were collected via visual inspection by plant pathologists of Northwest A\&F University. Rust assessment was conducted on 17/Apr/2018 (early onset of yellow rust, 25 days after rust inoculation) and 02/May/2018 (40 days after rust inoculation) for the upper-left $1 m \times 1 m$ square of each inoculated wheat plot. For each wheat plant in the $1 m \times 1 m$ square, yellow rust severity was estimated for three leaves including top, middle and lower ones 
Table 1: UAV survey details during the winter wheat growth season in 2017-2018, where Days (sow) means the days after sowing and GSD refers to the average ground sampling distance for the orthomosaic imagery.

\begin{tabular}{c|c|c|c|c}
\hline Date & Days (sow) & Stage & Altitude & GSD $(\mathrm{cm})$ \\
\hline $05 /$ Oct $/ 17$ & 0 & Seeding & NA & NA \\
\hline $11 /$ Dec/17 & 67 & Tillering & 25 & 1.65 \\
\hline $28 /$ Dec/17 & 84 & Overwintering & 25 & 1.54 \\
\hline $23 /$ Mar/18 & 169 & Stem extension & 20 & 1.19 \\
\hline $01 /$ Apr/18 & 178 & Stem extension & 15 & 1.03 \\
\hline $17 /$ Apr $/ 18$ & 194 & Stem extension & 20 & 1.42 \\
\hline $07 /$ May/18 & 214 & Heading & 15 & 1.07 \\
\hline $15 /$ May/18 & 222 & Heading & 20 & 1.21 \\
\hline $23 /$ May/18 & 230 & Ripening & 20 & 1.13 \\
\hline
\end{tabular}

following the Chinese yellow rust grade scale GB/T15795-2011 (see, left plot of Fig 4). A figure that shows the visual symptom of yellow rust disease is displayed in the right plot of Fig. 4

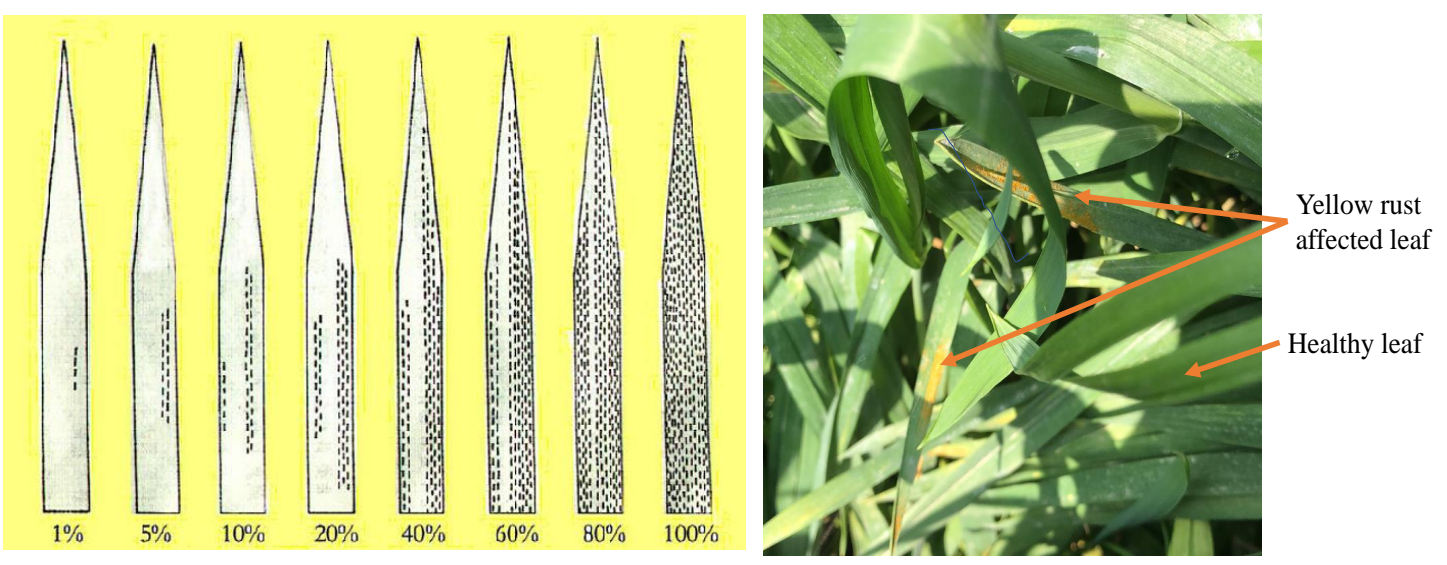

Figure 4: Left: grade scale of yellow rust severity [27]; Right: a phone-taken image showing the visual symptom of yellow rust disease.

The histograms along with trimean (i.e. mean excluding $5 \%$ outliers) for the yellow rust severity data of top leaves (on 17/Apr/2018 and 02/May/2018) are shown in Fig 5. The actual disease severity is consistent with the level of yellow rust inoculum received by the plot. The average disease severities for data on 17/Apr/2018 and 02/May/2018 are in the ranges of $[0.3 \%, 2 \%]$ and $[6.3 \%, 7.4 \%]$, respectively.

\section{Methods}

In this section, the methods are presented including spectral bands/indices calculation by Pix4DMapper software, wheat pixel extraction for yellow rust inoculated plots and mutual information for statistical dependency analysis. 

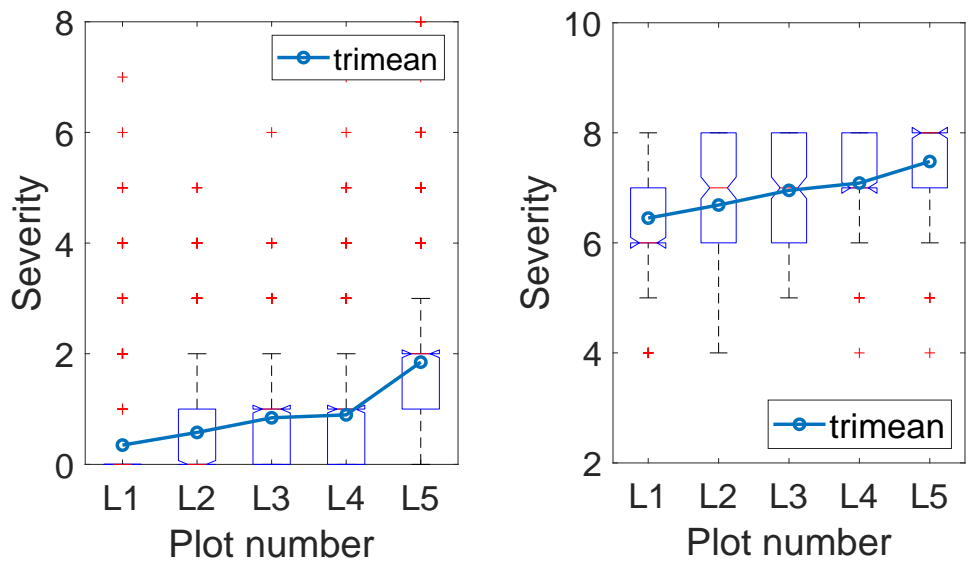

Figure 5: Ground severity data for wheat plots under various levels of yellow rust inoculum: 17/Apr/2018 (left) and 02/May/2018 (right), where L1-L5 denote Level 1-Level 5.

The overall workflow is displayed in Fig 6. In the following subsections, different components of the flowchart are elaborated.

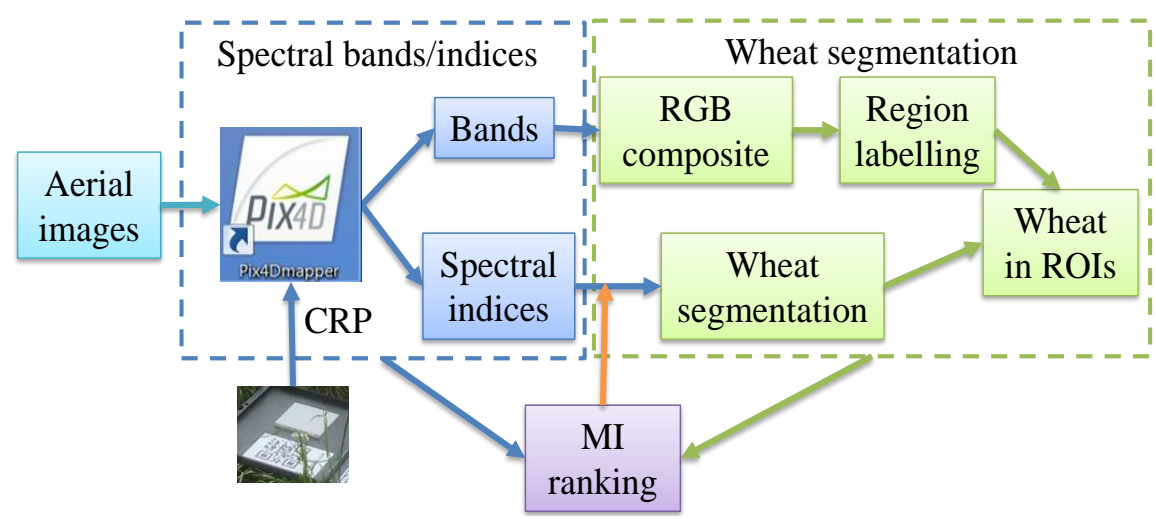

Figure 6: Overall flowchart including spectral bands/indices calculation, wheat pixel segmentation and Mutual Information (MI) based statistical dependency analysis.

\subsection{Spectral bands/indices calculation}

In this work, commercial photogrammetry software, Pix4DMapper of version 4.3.31 (Educational Licence of 1500 EUR, Pix4D SA, Switzerland), is adopted to process the raw images in order to generate orthomosaic images for calibrated bands and SVIs. The whole process includes initial processing (e.g. keypoint computation for image matching), Point Cloud and Mesh generation (optional) and orthomosaic generation and index calculation (with image calibration). In particular, in image calibration, both DLS for sun light information and the calibration panel images are used (please also refer to the preceding work [5, 4 for more details). Then spectral bands and indices maps are processed in Matlab 2017b/2018b for the follow-up analysis. SIs refer to various mathematical manipulations combining the reflectance data at two or three spectral bands [28] in order to enhance spectral differences. Adopting SI is a common technique in precision agriculture and has been applied in a wide range of areas such as weed mapping 
Table 2: A large number of SIs extracted from RedEdge five-bands multispectral imagery, where RE means RedEdge band. The formulae of SIs are referred to the preceding work 5

\begin{tabular}{|c|c|}
\hline Category & Name \& Reference \\
\hline Green-Red & Nitrogen Reflectance Index (NRI) [31, Greenness Index (GI) [16] \\
\hline Blue-Green-Red & $\begin{array}{l}\text { Green Leaf Index (GLI) [32, Normalized Excess Green (nexg) 33], Triangular } \\
\text { Greenness Index (TGI) 28] }\end{array}$ \\
\hline Green-RE & Anthocyanin Reflectance Index (ARI) [17] \\
\hline Green-NIR & $\begin{array}{l}\text { Green NDVI (GNDVI) 34, Triangular Vegetation Index (TVI), ChlorophyII } \\
\text { Index-Green (CIG) 35] }\end{array}$ \\
\hline Red-NIR & $\begin{array}{l}\text { Normalized Difference Vegetation Index (NDVI) [36], Soil Adjusted Vegetation } \\
\text { Index (SAVI) [37, Ratio Vegetation Index (RVI) [38, Optimized Soil Adjusted } \\
\text { Vegetation Index (OSAVI) 39] }\end{array}$ \\
\hline RE-NIR & $\begin{array}{l}\text { Normalized Difference RedEdge Index (NDREI) [40, ChlorophyII Index- } \\
\text { RedEdge (CIRE) } 35]\end{array}$ \\
\hline Blue-Red-NIR & Enhanced Vegetation Index (EVI) 41] \\
\hline Green-Red-RE & Transformed ChlorophyII Absorption and Reflectance Index (TCARI) 18] \\
\hline Green-Red-NIR & ChlorophyII Vegetation Index (CVI) 42 \\
\hline Red-RE-NIR & Simplified Canopy ChlorophyII Content Index (SCCCI) [43] \\
\hline
\end{tabular}

[29], yellow rust monitoring [5, yield estimation [30], wheat stress assessment [4]. On the basis of literature review and the available spectral bands on RedEdge multispectral camera, a large number of potential SIs are evaluated for estimating yellow rust. To make it more clear, these SIs are categorized according to the spectral bands adopted in index calculation in Table 2

\subsection{Wheat pixel segmentation for ROIs}

Wheat pixel segmentation is performed before conducting SI analysis for yellow rust, since the images contain both wheat and non-wheat pixels, which has adverse effects on reflectance data. It has also been shown in previous studies that removing soil background from images can substantially improve the results for SIs [4, 44]. Wheat's physical and chemical contents usually change over time, and consequently an effective index for one stage may not be useful for other stages. Therefore, a dedicated index for wheat segmentation should be selected for wheat at different stages.

With the aerial images by the UAV-Camera system in Section 2.2 image pre-processing was conducted by the method in Section 3.1 to derive the bands reflectance and SIs. Then with the reflectance data of Red, Green and 
Blue (RGB) bands, the RGB composite image over time for the wheat field with image adjustment for intensity enhancement is obtained (Fig 7). The wheat pixels in various inoculated plots are then extracted for an accurate

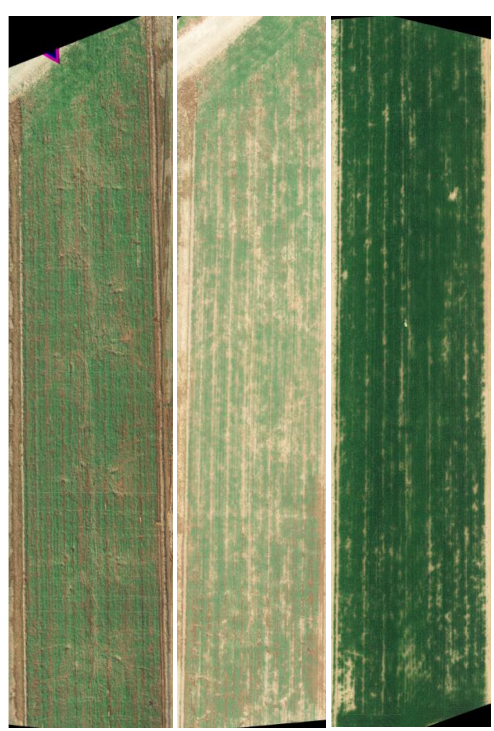

11/Dec/2017 28/Dec/2017 23/Mar/2018

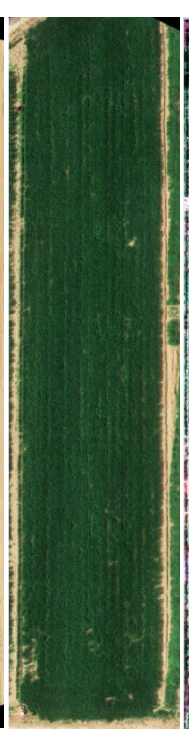

01/Apr/2018 17/Apr/2018
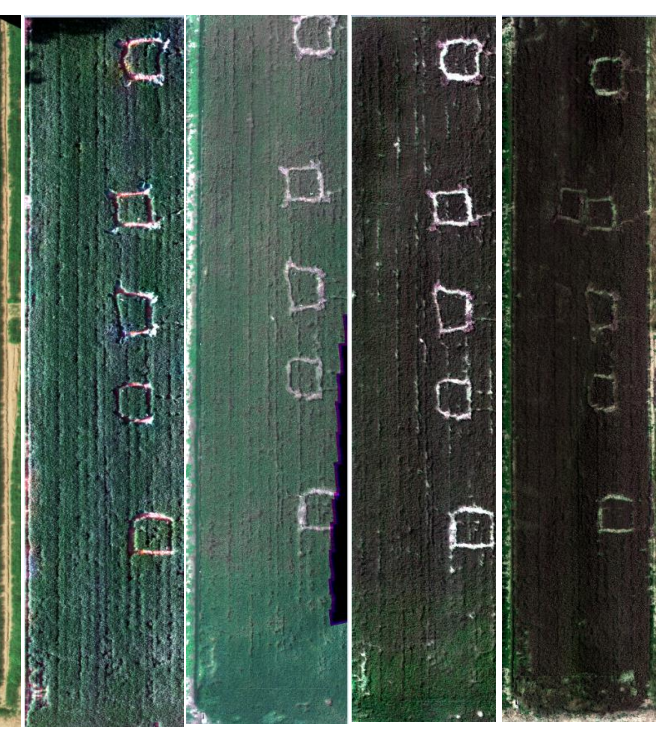

07/May/2018 15/May/2018 23/May/2018

Figure 7: RGB composite (by using band reflectance) over time of the wheat field with image intensity adjustment.

spectral analysis 23. This is achieved by three steps (Fig 6): 1. manually define the inoculated plot pixels in RGB composite image; 2 . extract the wheat pixels for the whole image; 3 . take the intersection of inoculated plot pixels and wheat pixels. The details are given as below. RGB composite of the orthomosaic image is generated in Matlab 2017b by layer-stacking the calibrated Red, Green and Blue bands, where Matlab command "imadjust" is adopted to adjust the image intensity (note: "imadjust" saturates the bottom $1 \%$ and the top $1 \%$ of all pixel values and therefore this operation can increase the contrast of the image). Then Matlab App "imageLabeler" is used to label various yellow rust inoculated wheat plots (termed inoculated plot pixels). As the labelled pixels in the aforementioned step by using region labelling may contain non-wheat pixels such as soil (especially in early stages due to a low canopy cover value [44]), wheat segmentation is further considered in the second step. There are a number of vegetation segmentation approaches (please refer to the survey paper [45]); a very popular method is the index-based approach due to its simplicity and effectiveness. In this approach, an index derived from spectral bands is defined to separate vegetation pixels from non-vegetation pixels, where the threshold is chosen either manually or by Otsu' algorithm [46].

Conventional indices for vegetation segmentation are mainly for RGB imagery [45], therefore, these indices are constrained to the combinations of visible bands (i.e. visible-indices). In particular, green vegetation shows relatively low values in Blue and Red bands while with a peak in Green band (i.e. Green peak phenomenon). Therefore, visibleindex NExG $=2 *$ Green-(Red+Blue) [33] is widely used in conventional studies [47]. It should be noted that: (i) there are five visible-infrared bands in this study and therefore more indices are available for vegetation segmentation; (ii) different indices should be used at different wheat growth (rust development) stages, since the wheat's physical and 
chemical contents change over time and as a result an useful index at one stage may become useless at other stages. Therefore, in this study, the indices for wheat segmentation at different stages are chosen automatically by analysing the temporal data.

In particular, some wheat pixels and non-wheat pixels are firstly labelled for the orthomosaic imageries at different stages, then statistical dependency analysis in Section 3.3 is drawn to evaluate the relationship between various SIs in Table 2 and wheat/non-wheat label. The SIs with the strongest statistical dependency are thus chosen as the segmentation index for each stage. Then the threshold segmenting wheat pixels from non-wheat pixels is manually determined by checking their corresponding index values. The wheat pixels in the yellow rust infected plots can be extracted by taking the intersection of inoculated plot pixels and wheat pixels. To make the aforementioned process comprehensive, wheat pixel segmentation for Region of Interest (ROIs) is summarized in Algorithm 1.

Algorithm 1: Wheat pixel segmentation for ROIs.

(a) Inoculated plot pixels: label various yellow rust inoculated wheat plots with RGB image composite and Matlab App "imageLabeler";

(b) Segmentation SIs: label wheat/non-wheat pixels for orthomosaic images at various wheat growth (rust development) stages; conduct statistical dependency analysis between SIs and wheat/non-wheat label; return the SIs with the strongest dependency as the segmentation indices;

(c) Wheat pixels: return wheat pixels by manually setting the threshold for segmentation SIs;

(d) Wheat pixels for ROIs: extract wheat pixels for ROIs by taking the intersection of inoculated plot pixels and wheat pixels.

\subsection{Statistical dependency analysis}

Statistical dependency analysis is then adopted to select SIs for segmenting wheat pixels from non-wheat pixels and also identify the effective bands/indices for yellow rust mapping. Mutual Information (MI) is preferred in this study due to its simplicity and effectiveness. MI, different from Pearson correlation measuring the linear relationship, measures the contribution of one variable (e.g. spectral bands/indices) towards reducing uncertainty about the value of another variable (e.g. wheat/non-wheat label, yellow rust label) [4]. For two discrete random variables $X, Y$ with $P(x), P(y), P(x, y)$ representing their marginal distributions and joint distribution, their MI value [48] is defined by

$$
M I(X, Y)=\sum_{x \in X} \sum_{y \in Y} P(x, y) \log \left(\frac{P(x, y)}{P(x) P(y)}\right) .
$$

It is noted that in the aforementioned calculation feature quantization is generally applied to discretize continuous variables into discrete bins [49. And a higher MI value implies a higher statistical dependency, and $M I(X ; Y)=0$ if 
and only if $X$ and $Y$ are independent random variables.

\section{Results}

In this section, the results are presented including wheat pixels extraction for yellow rust inoculated plots (Section 4.1), statistical dependency analysis between SIs and yellow rust severity label over time (Section 4.2), and spatiotemporal variations of sensitive bands/indices (Section 4.3).

\subsection{Wheat pixel extraction in ROIs}

Based on the RGB composite images, wheat squares of interest can be easily extracted via manual labelling with the "imageLabeler" in Matlab 2017b. Wheat segmentation is then considered. To select suitable segmentation indices at different stages, MI values between SIs and wheat/non-wheat label are calculated (Fig 8). It follows from Fig 8 that: (1) at a given stage, the segmentation abilities (reflected by the MI values) of various SIs are quite different; (2) the segmentation ability of a given index also varies at different stages. Therefore, the indices dedicated to particular stages should be selected, which are chosen as the ones with the strongest statistical dependency. The segmentation indices along with corresponding thresholds at various stages are summarized in Table 3 . It is also noted from Table 3 that even the same indices are selected for different stages, their segmentation thresholds change. The wheat pixel

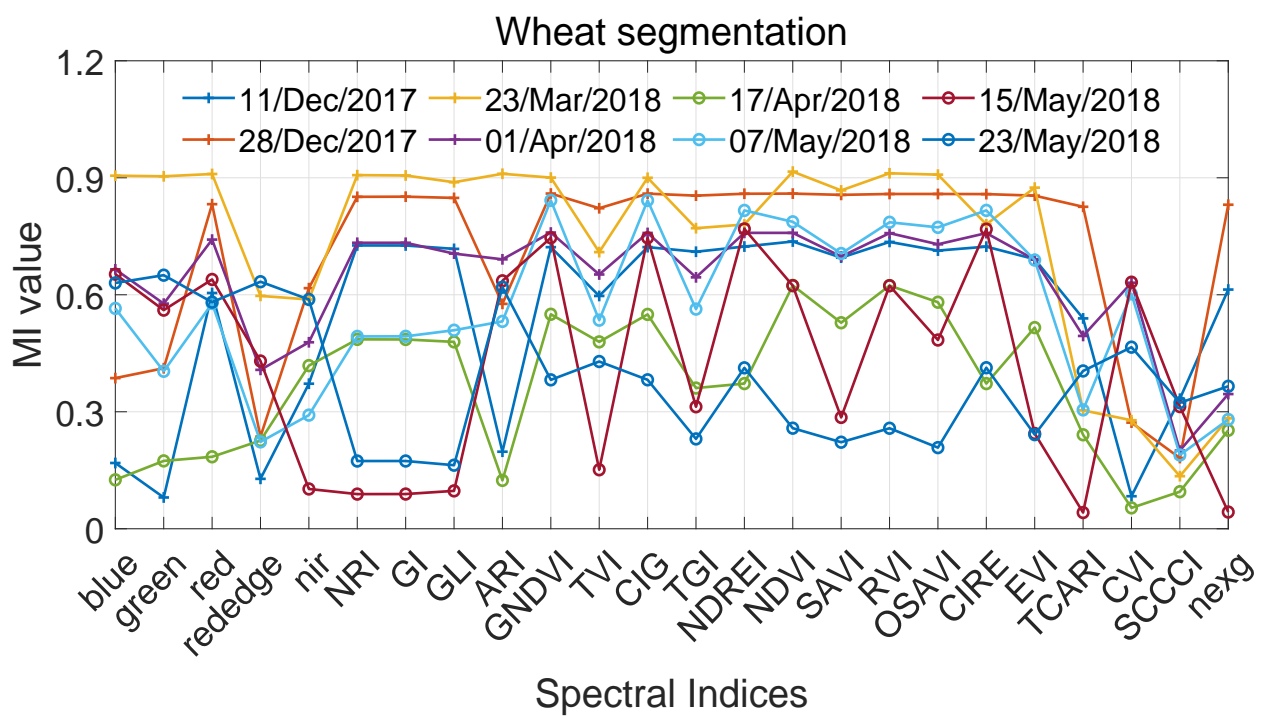

Figure 8: MI statistical values between bands/SIs and wheat/non-wheat label for wheat segmentation over the entire wheat growth season.

segmentation for the aerial imagery on $17 / \mathrm{Apr} / 2018$ is taken as an example, where the results for region labelling, wheat pixel segmentation and wheat pixels in plots of interest are shown in Fig 9 with "Un" being unlabelled pixels.

\subsection{SI analysis for yellow rust}

With wheat pixels in different squares being extracted, the statistical dependency between various bands/indices and labels for yellow rust disease is then evaluated over time by using MI approach in Section 3.3. A higher MI value 
Table 3: Segmentation indices with corresponding thresholds at different wheat growth (rust development) stages

\begin{tabular}{|c|c|c|c|}
\hline 11/Dec/2017 & 28/Dec/2017 & 23/Mar/2018 & 01/Apr/2018 \\
\hline $\operatorname{NDVI}(0.5)$ & $\operatorname{GNDVI}(0.44)$ & $\operatorname{NDVI}(0.8)$ & GNDVI(0.8) \\
\hline 17/Apr/2018 & 07/May/2018 & 15/May/2018 & 23/May/2018 \\
\hline $\operatorname{NDVI}(0.92)$ & GNDVI(0.52) & $\operatorname{NDREI}(0.22)$ & Green $(0.065)$ \\
\hline
\end{tabular}

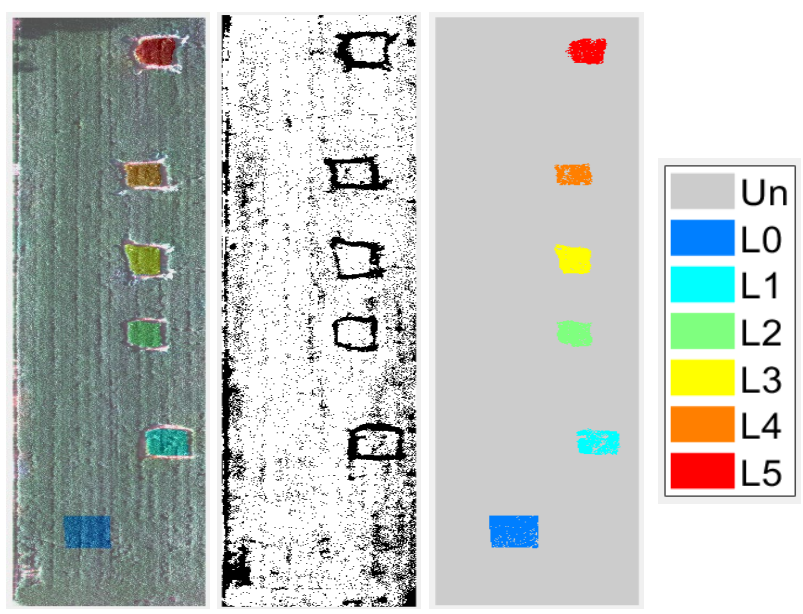

Figure 9: Region labelling overlay on RGB image (left), Wheat pixel segmentation by using NDVI index (middle) and their intersection as wheat pixel indices for wheat plots under various levels of yellow rust inoculum. Note: L0-L5 denote Level 0-Level 5.

means a stronger statistical dependency and therefore a better discriminating ability. The continuous bands/indices are quantized into 12 discrete bins for MI calculation. The comparative results are shown in Fig. 10 . The following observations can be drawn from Fig 10.

(1) Before yellow rust inoculation (23/Mar/2018, 169 days after sowing), all spectral bands/indices have very low MI values (i.e. lower than 0.1). This is because all wheat squares are healthy without yellow rust lesions yet.

(2) On 23/Mar/2018 (inoculation date), 01/Apr/2018 (rust incubation stage, 9 days after inoculation) and 17/Apr/2018 (early onset, 25 days after inoculation), the MI values are still very small (lower than 0.1). This means that at inoculation, incubation and early onset stages, it is not possible to rely on a single spectral band or index to monitor the status of yellow rust, although as shown in the left plot of Fig 5 visible yellow rust symptom has already occurred at a severity level of $2 \%$ via ground leaf assessment on 17/Apr/2018.

(3) At the disease development stages (e.g. 07/May/2018, 15/May/2018, 23/May/2018), a number of spectral bands and indices have high statistical dependencies with yellow rust severity level. Moreover, the level of statistical dependency changes over time. For example, on 05/May/2018, the bands and indices having strong statistical dependency are Red/NIR and NDVI/RVI, which are NIR and OSAVI on 15/May/2018. This means that the selection of sensitive spectral bands/indices should take the disease development stage into account. 


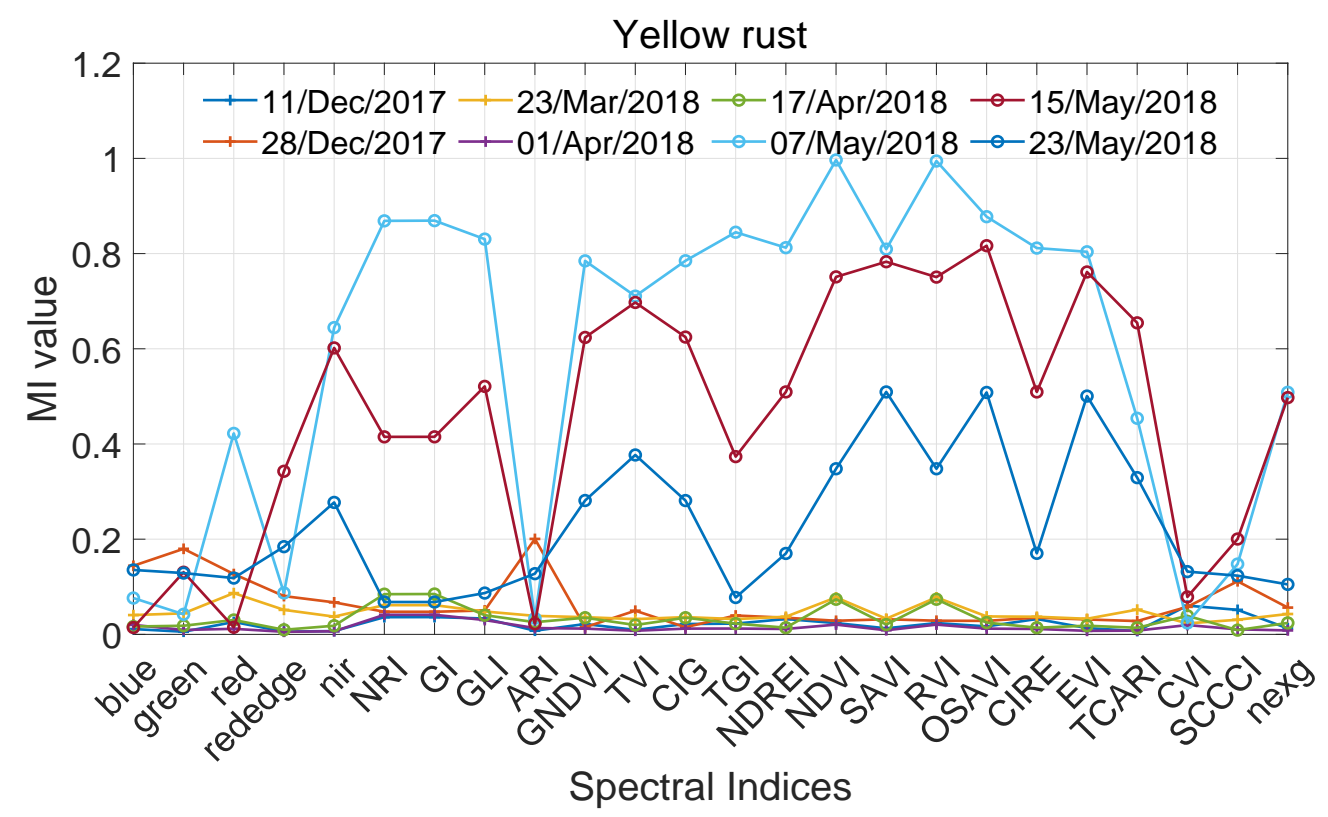

Figure 10: MI statistical dependency values between bands/SIs and yellow rust labels for eight time points covering the wheat growth season.

(4) By taking the component bands of various SIs in Table 2 into account, overall, the SIs (e.g. NDVI, SAVI, RVI, OSAVI) comprised of Red and NIR bands generally outperform other indices. The changes in Red are mainly due to the changes of wheat color induced by the pustules containing thousands of dry yellow-orange to reddish-brown spores. While the changes in NIR reflects the internal changes of leaf structure induced by yellow rust disease.

\subsection{Spatio-temporal variations of sensitive bands/indices}

It follows from Fig 10 that the sensitive bands for yellow rust are Red and NIR, and the sensitive indices are also derived by these two bands (e.g. NDVI, SAVI, RVI, OSAVI). In this section, their temporal variations (note: NDVI index is chosen as the sensitive index for the purpose of illustration) for wheat squares inoculated with various levels of yellow rust inoculum are investigated quantitatively. Their trimean values over different survey time points covering the wheat growth season are illustrated in Figs 11 and 12 The following observations can be drawn from Figs 11 and 12 .

(i) On 18/Dec/2017 (84 days after sowing), an obvious increase and a slight decrease are observed in Red/NIR reflectance and NDVI index in comparison to the ones on 11/Dec/2017 (67 days after sowing). The main reason is that irrigation has been conducted on 10/Dec/2018 (so the moisture level is expected to increase on $11 / \mathrm{Dec} / 2018$ ) and the wheat is in the early stage of overwintering on 28/Dec/2018 (so the wheat vigor is expected to be lower). This can also be observed by the RGB image in Fig 7 . 

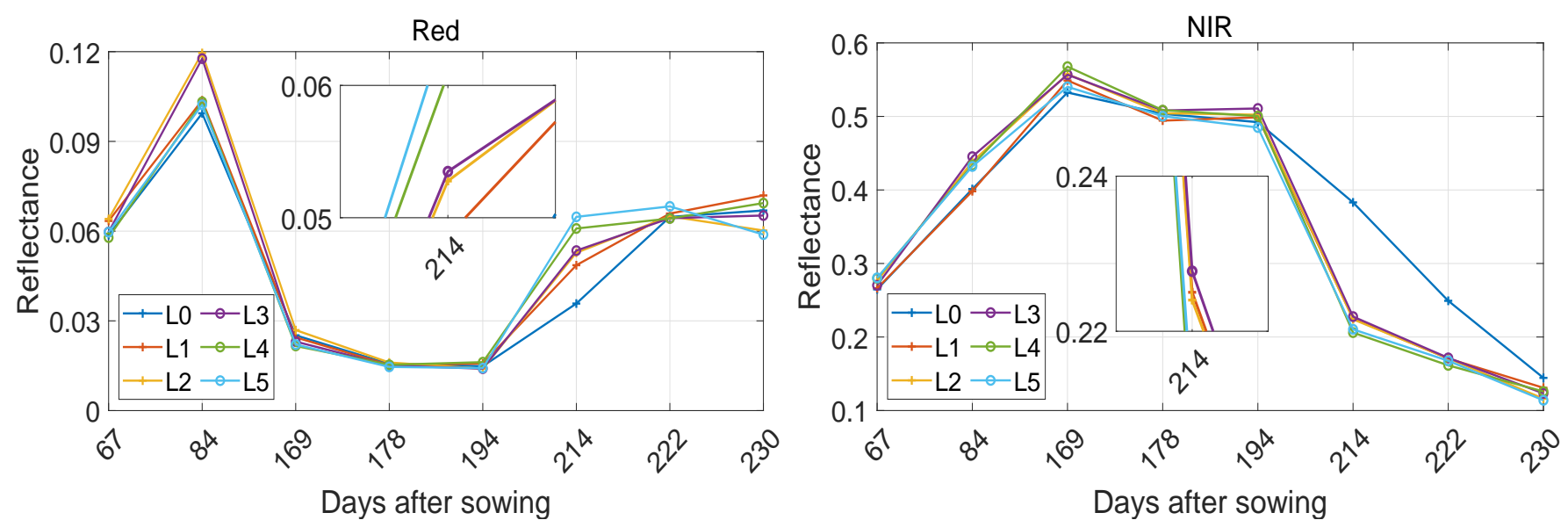

Figure 11: Temporal variations of Red and NIR band reflectance for wheat plots with various levels of yellow rust inoculum.

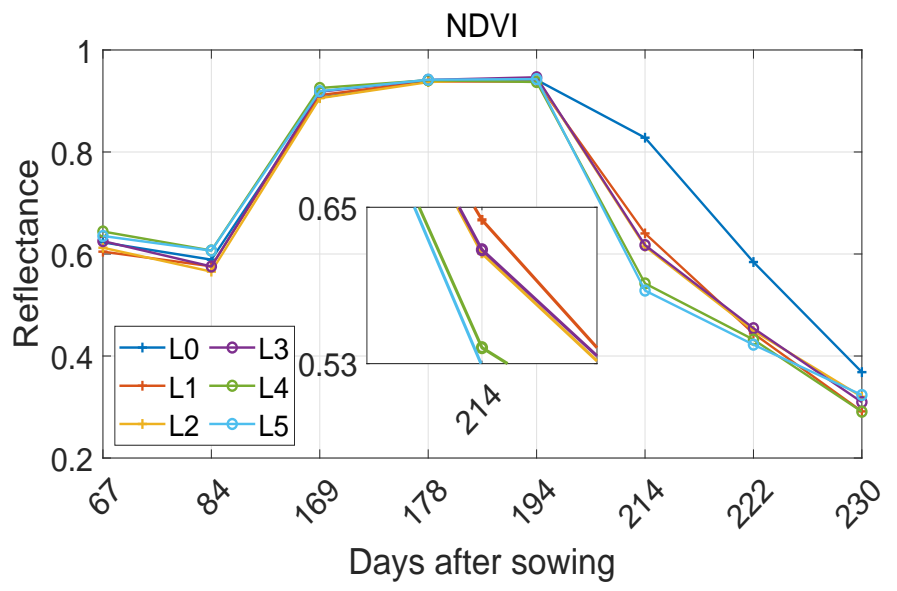

Figure 12: NDVI temporal variations in various wheat plots.

(ii) From 23/Mar/2018 (169 days after sowing) to 17/Apr/2018 (194 days after sowing), the reflectance of Red (low) and NIR (high) bands become relatively stable, and therefore the phenomenon of NDVI saturation (at a value of 0.9 ) has been observed in Fig 12. More importantly, although visible yellow rust symptoms have already been observed on ground on the top wheat leaf at a low level of 2\% on 17/Apr/2018 (194 days after sowing and 25 days after yellow rust inoculation), no reflectance changes are observed in Red/NIR bands or SIs including NDVI.

(iii) However, significant changes are observed in Red (an increased reflectance), NIR (a decreased reflectance) and NDVI (a decreased value) on 07/May/2018 (214 days after sowing and 45 days after rust inoculation). The changes among wheat squares inoculated by different levels of yellow rust inoculum are different and consistent with the initial levels of inoculum, which facilitates selecting suitable SIs (e.g. NDVI) for yellow rust severity monitoring.

(iv) The NDVI value keeps decreasing in the late Heading and the Ripening stages (e.g. on 15/May/2018 and 23/May/2018), along with this trend the differences among wheat plots inoculated with various levels of yellow 
rust inoculum become weaken, which makes the yellow rust severity monitoring more difficult. It can also be observed that on 23/May/2018 (230 days after sowing and 61 days after inoculation) there is no significant difference in NDVI value between healthy and yellow rust infected wheat pixels any more.

In addition to the temporal variations of different sensitive bands/indices, it would also be worthwhile to show their spatial distribution. The spatio-temporal variation of NDVI index is taken as an example (Fig 13), where a brighter pixel means a higher NDVI value. Some aforementioned observations (e.g. observations ii iii and iv) can also be visually seen in Fig 13. In addition, NDVI difference can also be observed in various yellow rust infected wheat plots, particularly for the data on $07 / \mathrm{May} / 2018$.

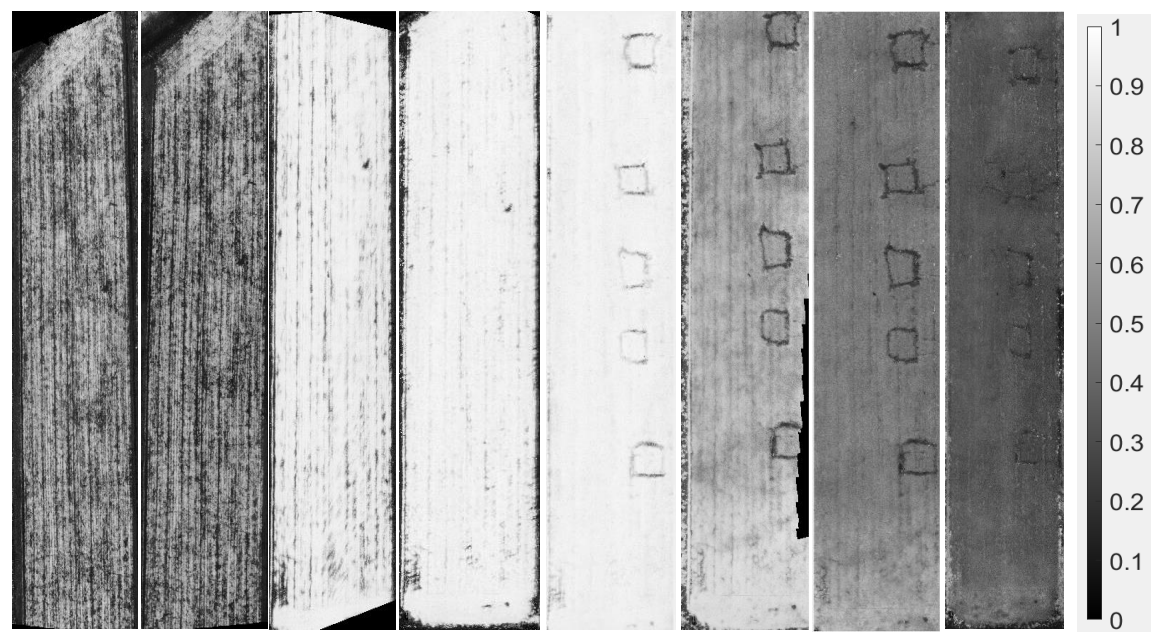

Figure 13: Spatio-temporal variations of NDVI index for the experimental wheat field including different wheat plots inoculated with various levels of yellow rust inoculum.

\section{Discussions}

Wheat segmentation: Regarding vegetation segmentation, the classical and widely-used visible-index NExG [33], relying on the Green peak phenomenon, is not effective at a later growth stage. This is because as shown in Fig 14 the Green peak phenomenon no longer holds at a later growth stage (23/May/2018), although it is valid at an early growth stage (23/Mar/2018), i.e. green reflectance of wheat pixels are higher than blue and red. Therefore, various segmentation indices are selected in this study, which are dedicated to wheat growth (rust development) stages, leading to satisfying results. It should also be highlighted that wheat segmentation is not easy for imaging systems with a poor resolution such as hyperspectral camera on-board an UAV [50] or aircraft/satellite remote sensing.

Spatio-temporal monitoring: Different from many existing studies on UAV remote sensing for yellow rust (or other diseases) monitoring where one-time monitoring is concerned [5, 23, in this work, an efficient workflow is devised to dynamically monitor wheat yellow rust development over the entire wheat growth season. As a result, dedicated 


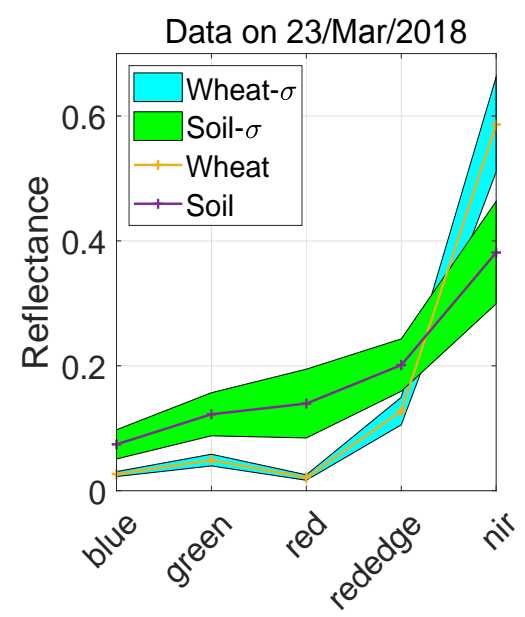

Bands

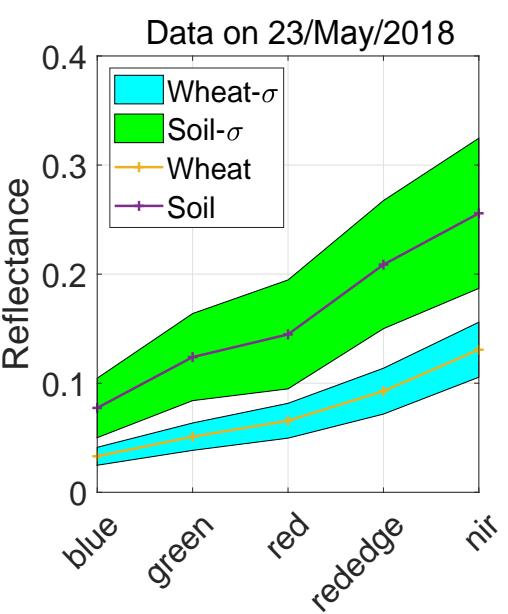

Bands

Figure 14: Reflectance (mean and $1-\sigma$ region) data for wheat and soil pixels on 23/Mar/2018 and 23/May/2018, respectively.

indices are selected for wheat segmentation at different stages and the effectiveness of spectral bands/indices for yellow rust are not only assessed via statistical dependency analysis but also dynamically and quantitatively analysed. This is more desirable for the early and quantitative monitoring of crop [24, 4, 25] including wheat yellow rust in this study.

Bands/Indices changes: It is discovered that Red and NIR are effective spectral bands in the late disease stages, however, no spectral bands are effective for rust disease assessment at the incubation stage (9 days after inoculation) and early onset stage (25 days after inoculation, with a relatively low disease severity of $2 \%$ ). This highlights the challenges of early yellow rust disease monitoring by using the current UAV-camera remote sensing systems. It is also discovered that yellow rust disease leads to an increase in Red and a decrease in NIR reflectance; and more importantly the amount of reflectance's increase or decrease is almost consistent with the level of yellow rust inoculation (or disease severity). This is mainly because yellow rust disease leads to a reduction of ChlorophyII content and an increased evaporation (i.e. an increased water loss or even yellow color on wheat leaves at late stages), which can be effectively captured by multispectral images. Statistical dependency analysis via mutual information reveals that the normalized difference between NIR and Red bands (i.e. the spectral index NDVI) is more effective than either NIR or Red band. Considering that other stresses (e.g. nitrogen [51], water stress [52], etc.) may also have effects on NDVI values, the main variables to be inferred should be determined beforehand [4] and more background information (e.g. climate information, growth stage information) should also be taken into account in real-life agriculture applications. In addition, time-series measurement information should also be carefully analysed, since valuable information can be inferred such as the way in which the NDVI changes over time (i.e. trend information).

Disease early detection: It is generally challenging to detect yellow rust disease in early stages (e.g. incubation stages) by using remote sensing approaches [5]. The possible reasons in this work are summarized as below. Firstly, the multispectral image adopted in this work possesses five bands in the spectral range of visible and NIR spectrum, 
so the first reason may be due to the limited spectral range (i.e. short spectral range). Secondly, the spatial resolution of the multispectral image in this work is over $1 \mathrm{~cm}$, therefore, the subtle changes in small diseased areas may not be effectively captured by the images due to a low spatial resolution (i.e. low spatial resolution). Thirdly, the spectral resolution of the multispectral image is at $20 \mathrm{~nm}, 10 \mathrm{~nm}$ and $40 \mathrm{~nm}$ respectively, which is far lower than that of hyperspectral image (i.e. low spatial resolution). Therefore, corresponding measures should be taken in order to address the problem of disease early detection.

Future research: Although a crop disease monitoring system is developed with a multispectral camera and an UAV, and its performance has been validated by the spatio-temporal monitoring of wheat yellow rust during the entire wheat growth season, there is still much room for further development. Some key aspects are summarized as follows:

(1) More frequent surveys will be performed in early disease stages so that the capability of UAV remote sensing for early disease detection can be evaluated more accurately;

(2) A quantitative relationship between UAV remote sensing information (e.g. NDVI) and yellow rust disease severity (e.g. ground severity label) will be established quantitatively, which can be achieved by designing morel levels of yellow rust inoculum and taking the severity information over time into account;

(3) Hyperspectral camera on-board an UAV will be investigated for yellow rust monitoring in the incubation and early lesion onset stages.

\section{Conclusions}

This work develops a dedicated workflow comprised of an UAV and a multispectral camera to monitor wheat yellow rust disease both spatially and temporally during the entire growth season. A wheat yellow rust experiment is designed by inoculating wheat plots with one of the six levels of yellow rust inoculum including an un-inoculated control. Aerial five-bands multispectral images are obtained at eight key time points covering the entire wheat growth season with the UAV-Camera system at an altitude of about 20 meters. Statistical dependency analysis via mutual information is used to select the sensitive bands/indices for both wheat segmentation and yellow rust severity estimation. Temporal analysis shows that various indices for wheat segmentation should be used to account for wheat canopy changes at different stages. Red and NIR are the effective bands for yellow rust disease and their normalized difference NDVI index provides a even better result in disease stages (early and middle). Some challenges have also been observed in current UAV remote sensing system for the early monitoring of yellow rust disease, where no bands/indices are sensitive at the incubation stage ( 9 days after inoculation) and early onset (25 days after inoculation with a disease severity of $2 \%$ on top wheat leaves). Future research for early disease detection of yellow rust is also discussed. Although the 
system and workflow devised in this work are for yellow rust monitoring, it can find a wide ranges of applications (i.e. drought, grass, nitrogen, biomass and pest assessment) in precision agriculture where spatial-temporal monitoring is involved.

\section{Contributions}

Project idea (Wen-Hua Chen, Xiangming Xu, Cunjia Liu, Lei Guo, Jinya Su); Experiment design (Cunjia Liu, Xiaoping $\mathrm{Hu}$, Xiangming $\mathrm{Xu}$, and Jinya Su); UAV-Camera system (Cunjia Liu and Jinya Su); Ground data collection (Xiaoping $\mathrm{Hu}$ ); Data processing, algorithm design and implementation (Jinya Su); Paper writing (Jinya Su, Wen-Hua Chen, Xiangming Xu), Coordination (Lei Guo, Xiangming Xu, Cunjia Liu and Jinya Su).

\section{Acknowledgements}

This work was supported by Science and Technology Facilities Council (STFC) under Newton fund with Grant No. ST/N006852/1. Prof. Xiaoping Hu was supported by National Natural Science Foundation of China with Grant No. 31772102. Dr. Matthew Coombes was acknowledged for developing the UAV-Camera system; Xi'an Tongfei Aviation Technology Co., Ltd was acknowledged for their professional support in flying UAV for data collection; team members (e.g. Mr. Conghao Wang) of Prof. Xiaoping Hu were acknowledged for ground yellow rust data collection.

\section{References}

[1] L. Yuan, Y. Huang, R. W. Loraamm, C. Nie, J. Wang, and J. Zhang, "Spectral analysis of winter wheat leaves for detection and differentiation of diseases and insects," Field Crops Research, vol. 156, pp. 199-207, 2014.

[2] D. Moshou, C. Bravo, J. West, S. Wahlen, A. McCartney, and H. Ramon, "Automatic detection of yellow rustin wheat using reflectance measurements and neural networks," Computers and electronics in agriculture, vol. 44, no. 3, pp. 173-188, 2004.

[3] S. Sankaran, A. Mishra, R. Ehsani, and C. Davis, "A review of advanced techniques for detecting plant diseases," Computers and Electronics in Agriculture, vol. 72, no. 1, pp. 1-13, 2010.

[4] T. Duan, S. Chapman, Y. Guo, and B. Zheng, "Dynamic monitoring of ndvi in wheat agronomy and breeding trials using an unmanned aerial vehicle," Field Crops Research, vol. 210, pp. 71-80, 2017.

[5] J. Su, C. Liu, M. Coombes, X. Hu, C. Wang, X. Xu, Q. Li, L. Guo, and W.-H. Chen, "Wheat yellow rust monitoring by learning from multispectral uav aerial imagery," Computers and electronics in agriculture, vol. 155, pp. 157-166, 2018.

[6] A. De Castro, R. Ehsani, R. Ploetz, J. Crane, and J. Abdulridha, "Optimum spectral and geometric parameters for early detection of laurel wilt disease in avocado," Remote Sensing of Environment, vol. 171, pp. 33-44, 2015. 
[7] C. Zhang and J. M. Kovacs, "The application of small unmanned aerial systems for precision agriculture: a review," Precision agriculture, vol. 13, no. 6, pp. 693-712, 2012.

[8] J. Zhang, R. Pu, R. W. Loraamm, G. Yang, J. Wang, et al., "Comparison between wavelet spectral features and conventional spectral features in detecting yellow rust for winter wheat," Computers and Electronics in Agriculture, vol. 100, pp. 79-87, 2014.

[9] N. Wouters, B. De Ketelaere, J. De Baerdemaeker, and W. Saeys, "Hyperspectral waveband selection for automatic detection of floral pear buds," Precision agriculture, vol. 14, no. 1, pp. 86-98, 2013.

[10] S. Sankaran, A. Mishra, J. M. Maja, and R. Ehsani, "Visible-near infrared spectroscopy for detection of huanglongbing in citrus orchards," Computers and electronics in agriculture, vol. 77, no. 2, pp. 127-134, 2011.

[11] R. Devadas, D. Lamb, S. Simpfendorfer, and D. Backhouse, "Evaluating ten spectral vegetation indices for identifying rust infection in individual wheat leaves," Precision Agriculture, vol. 10, no. 6, pp. 459-470, 2009.

[12] R. A. Naidu, E. M. Perry, F. J. Pierce, and T. Mekuria, "The potential of spectral reflectance technique for the detection of grapevine leafroll-associated virus-3 in two red-berried wine grape cultivars," Computers and Electronics in Agriculture, vol. 66 , no. 1, pp. 38-45, 2009.

[13] N. Bagheri, H. Mohamadi-Monavar, A. Azizi, and A. Ghasemi, "Detection of fire blight disease in pear trees by hyperspectral data," European Journal of Remote Sensing, vol. 51, no. 1, pp. 1-10, 2018.

[14] D. Ashourloo, M. R. Mobasheri, and A. Huete, "Evaluating the effect of different wheat rust disease symptoms on vegetation indices using hyperspectral measurements," Remote Sensing, vol. 6, no. 6, pp. 5107-5123, 2014.

[15] P. S. Thenkabail, R. B. Smith, and E. De Pauw, "Hyperspectral vegetation indices and their relationships with agricultural crop characteristics," Remote sensing of Environment, vol. 71, no. 2, pp. 158-182, 2000.

[16] P. J. Zarco-Tejada, A. Berjón, R. López-Lozano, J. R. Miller, P. Martín, V. Cachorro, M. González, and A. De Frutos, "Assessing vineyard condition with hyperspectral indices: Leaf and canopy reflectance simulation in a row-structured discontinuous canopy," Remote Sensing of Environment, vol. 99, no. 3, pp. 271-287, 2005.

[17] A. A. Gitelson, M. N. Merzlyak, and O. B. Chivkunova, "Optical properties and nondestructive estimation of anthocyanin content in plant leaves," Photochemistry and photobiology, vol. 74, no. 1, pp. 38-45, 2001.

[18] D. Haboudane, J. R. Miller, N. Tremblay, P. J. Zarco-Tejada, and L. Dextraze, "Integrated narrow-band vegetation indices for prediction of crop chlorophyll content for application to precision agriculture," Remote sensing of environment, vol. 81, no. 2-3, pp. 416-426, 2002. 
[19] W. Huang, Q. Guan, J. Luo, J. Zhang, J. Zhao, D. Liang, L. Huang, and D. Zhang, "New optimized spectral indices for identifying and monitoring winter wheat diseases," IEEE Journal of Selected Topics in Applied Earth Observations and Remote Sensing, vol. 7, no. 6, pp. 2516-2524, 2014.

[20] W. Huang, D. W. Lamb, Z. Niu, Y. Zhang, L. Liu, and J. Wang, "Identification of yellow rust in wheat using in-situ spectral reflectance measurements and airborne hyperspectral imaging," Precision Agriculture, vol. 8, no. 4-5, pp. 187-197, 2007.

[21] J. Zhang, W. Huang, J. Li, G. Yang, J. Luo, X. Gu, and J. Wang, "Development, evaluation and application of a spectral knowledge base to detect yellow rust in winter wheat," Precision Agriculture, vol. 12, no. 5, pp. 716-731, 2011.

[22] L. Yuan, H. Zhang, Y. Zhang, C. Xing, and Z. Bao, "Feasibility assessment of multi-spectral satellite sensors in monitoring and discriminating wheat diseases and insects," Optik-International Journal for Light and Electron Optics, vol. 131, pp. 598$608,2017$.

[23] W. Liu, G. Yang, F. Xu, H. Qiao, J. Fan, Y. Song, and Y. Zhou, "Comparisons of detection of wheat stripe rust using hyperspectral and uav aerial photography," Acta Phytopathologica Sinica, vol. 48, no. 2, pp. 223-227, 2018.

[24] M. A. Hassan, M. Yang, A. Rasheed, G. Yang, M. Reynolds, X. Xia, Y. Xiao, and Z. He, "A rapid monitoring of ndvi across the wheat growth cycle for grain yield prediction using a multi-spectral uav platform," Plant Science, 2018.

[25] M. A. Hassan, M. Yang, A. Rasheed, X. Jin, X. Xia, Y. Xiao, and Z. He, "Time-series multispectral indices from unmanned aerial vehicle imagery reveal senescence rate in bread wheat," Remote Sensing, vol. 10, no. 6, p. 809, 2018.

[26] L. G. T. Crusiol, J. d. F. C. Carvalho, R. N. R. Sibaldelli, W. Neiverth, A. do Rio, L. C. Ferreira, S. de Oliveira Procópio, L. M. Mertz-Henning, A. L. Nepomuceno, N. Neumaier, et al., "Ndvi variation according to the time of measurement, sampling size, positioning of sensor and water regime in different soybean cultivars," Precision Agriculture, vol. 18, no. 4, pp. $470-490,2017$.

[27] "http://www.sdtdata.com/fx/fmoa/tslibcard.doview," Accessed on 20/11/2018.

[28] E. R. Hunt Jr, P. C. Doraiswamy, J. E. McMurtrey, C. S. Daughtry, E. M. Perry, and B. Akhmedov, "A visible band index for remote sensing leaf chlorophyll content at the canopy scale," International Journal of Applied Earth Observation and Geoinformation, vol. 21, pp. 103-112, 2013.

[29] M. Pérez-Ortiz, J. Peña, P. A. Gutiérrez, J. Torres-Sánchez, C. Hervás-Martínez, and F. López-Granados, "A semisupervised system for weed mapping in sunflower crops using unmanned aerial vehicles and a crop row detection method," Applied Soft Computing, vol. 37, pp. 533-544, 2015.

[30] T. S. Magney, J. U. Eitel, D. R. Huggins, and L. A. Vierling, "Proximal ndvi derived phenology improves in-season predictions of wheat quantity and quality," Agricultural and Forest Meteorology, vol. 217, pp. 46-60, 2016. 
[31] I. Filella, L. Serrano, J. Serra, and J. Penuelas, "Evaluating wheat nitrogen status with canopy reflectance indices and discriminant analysis," Crop Science, vol. 35, no. 5, pp. 1400-1405, 1995.

[32] M. Louhaichi, M. M. Borman, and D. E. Johnson, "Spatially located platform and aerial photography for documentation of grazing impacts on wheat," Geocarto International, vol. 16, no. 1, pp. 65-70, 2001.

[33] D. M. Woebbecke, G. E. Meyer, K. Von Bargen, and D. Mortensen, "Color indices for weed identification under various soil, residue, and lighting conditions," Transactions of the ASAE, vol. 38, no. 1, pp. 259-269, 1995.

[34] A. A. Gitelson, Y. J. Kaufman, and M. N. Merzlyak, "Use of a green channel in remote sensing of global vegetation from eos-modis," Remote sensing of Environment, vol. 58, no. 3, pp. 289-298, 1996.

[35] A. A. Gitelson, Y. Gritz, and M. N. Merzlyak, "Relationships between leaf chlorophyll content and spectral reflectance and algorithms for non-destructive chlorophyll assessment in higher plant leaves," Journal of plant physiology, vol. 160, no. 3, pp. 271-282, 2003.

[36] J. Rouse Jr, R. Haas, J. Schell, and D. Deering, "Monitoring vegetation systems in the great plains with erts," 1974.

[37] A. R. Huete, "A soil-adjusted vegetation index (savi)," Remote sensing of environment, vol. 25, no. 3, pp. 295-309, 1988.

[38] R. L. Pearson and L. D. Miller, "Remote mapping of standing crop biomass for estimation of the productivity of the shortgrass prairie," in Remote Sensing of Environment, VIII, p. 1355, 1972.

[39] G. Rondeaux, M. Steven, and F. Baret, "Optimization of soil-adjusted vegetation indices," Remote sensing of environment, vol. 55, no. 2, pp. 95-107, 1996.

[40] A. Gitelson and M. N. Merzlyak, "Quantitative estimation of chlorophyll-a using reflectance spectra: Experiments with autumn chestnut and maple leaves," Journal of Photochemistry and Photobiology B: Biology, vol. 22, no. 3, pp. 247-252, 1994.

[41] A. Huete, K. Didan, T. Miura, E. P. Rodriguez, X. Gao, and L. G. Ferreira, "Overview of the radiometric and biophysical performance of the modis vegetation indices," Remote sensing of environment, vol. 83, no. 1-2, pp. 195-213, 2002.

[42] M. Vincini, E. Frazzi, and P. DAlessio, "A broad-band leaf chlorophyll vegetation index at the canopy scale," Precision Agriculture, vol. 9, no. 5, pp. 303-319, 2008.

[43] T. Raper and J. Varco, "Canopy-scale wavelength and vegetative index sensitivities to cotton growth parameters and nitrogen status," Precision Agriculture, vol. 16, no. 1, pp. 62-76, 2015.

[44] J. Torres-Sánchez, J. M. Peña, A. I. de Castro, and F. López-Granados, "Multi-temporal mapping of the vegetation fraction in early-season wheat fields using images from uav," Computers and Electronics in Agriculture, vol. 103, pp. 104-113, 2014. 
[45] E. Hamuda, M. Glavin, and E. Jones, "A survey of image processing techniques for plant extraction and segmentation in the field," Computers and Electronics in Agriculture, vol. 125, pp. 184-199, 2016.

[46] N. Otsu, "A threshold selection method from gray-level histograms," IEEE transactions on systems, man, and cybernetics, vol. 9, no. 1, pp. 62-66, 1979.

[47] W. Guo, U. K. Rage, and S. Ninomiya, "Illumination invariant segmentation of vegetation for time series wheat images based on decision tree model," Computers and electronics in agriculture, vol. 96, pp. 58-66, 2013.

[48] T. M. Cover and J. A. Thomas, Elements of information theory. John Wiley \& Sons, 2012.

[49] J. Su, D. Yi, C. Liu, L. Guo, and W.-H. Chen, "Dimension reduction aided hyperspectral image classification with a small-sized training dataset: experimental comparisons," Sensors, vol. 17, no. 12, p. 2726, 2017.

[50] J. Torres-Sánchez, F. López-Granados, A. I. De Castro, and J. M. Peña-Barragán, "Configuration and specifications of an unmanned aerial vehicle (uav) for early site specific weed management," PloS one, vol. 8, no. 3, p. e58210, 2013.

[51] K. Erdle, B. Mistele, and U. Schmidhalter, "Comparison of active and passive spectral sensors in discriminating biomass parameters and nitrogen status in wheat cultivars," Field Crops Research, vol. 124, no. 1, pp. 74-84, 2011.

[52] J. Gago, C. Douthe, R. Coopman, P. Gallego, M. Ribas-Carbo, J. Flexas, J. Escalona, and H. Medrano, "Uavs challenge to assess water stress for sustainable agriculture," Agricultural water management, vol. 153, pp. 9-19, 2015. 